EPJ Web of Conferences 66, 10004 (2014)

DOI: 10.1051/epjconf/20146610004

(C) Owned by the authors, published by EDP Sciences, 2014

\title{
Fragmentation cross sections at intermediate energies for hadrontherapy and space radiation protection
}

\author{
M. De Napoli ${ }^{1, a}$, C. Agodi ${ }^{2}$, M. Bondi ${ }^{2}$, F. Cappuzzello 2,3 , D. Carbone ${ }^{2}$, M. Cavallaro², G.A.P. \\ Cirrone $^{2}$, G. Cuttone ${ }^{2}$, D. Nicolosi ${ }^{2,3}$, L. Pandola ${ }^{4}$, G. Raciti ${ }^{2,3, b}$, F. Romano ${ }^{2,3}$, D. Sardina ${ }^{2}$, V. \\ Scuderi ${ }^{2,5}$, and S. Tropea ${ }^{2,3}$ \\ ${ }^{1}$ Istituto Nazionale di Fisica Nucleare, Sezione di Catania, Via S. Sofia 6495123 Catania, Italy \\ ${ }^{2}$ Istituto Nazionale di Fisica Nucleare, Laboratori Nazionali del Sud, Via S. Sofia 6295123 Catania, Italy \\ ${ }^{3}$ Dipartimento di Fisica e Astronomia, Universita degli Studi di Catania, 64, Via S. Sofia, I-95123 Catania, \\ Italy \\ ${ }^{4}$ Istituto Nazionale di Fisica Nucleare,Laboratori Nazionali del Gran Sasso,SS 17 bis km 18+910, I-67100 \\ Assergi (AQ), Italy \\ ${ }^{5}$ Institute of Physics of the ASCR, Ceskomoravska 2420/15a, 19093 Prague, Czech Republic
}

\begin{abstract}
Nuclear fragmentation studies, historically driven by the interest of the nuclear physicists, are nowadays the subject of great attention for the hadrontherapy and the space radiation protection communities. In both fields, fragmentation cross sections are needed to predict the effects of the ion nuclear interactions within the patient's and the astronaut's body. Indeed, the the Monte Carlo codes used in planning tumor treatments and space missions must be tuned and validated by experimental data. However, only a limited set of fragmentation cross sections are available in literature, especially at Fermi energies. Therefore we have studied the production of secondary fragments in the ${ }^{12} \mathrm{C}+{ }^{12} \mathrm{C}$ and ${ }^{12} \mathrm{C}+{ }^{197} \mathrm{Au}$ reactions at $62 \mathrm{AMeV}$. Some of the measured cross sections are presented in this work.
\end{abstract}

\section{Introduction}

The purpose of any radiotherapy treatment is to maximize the damage to the tumor while preserving, as much as possible, the surrounding healthy tissues. In this respect, the use of carbon ion beams in hadrontherapy presents many physical and biological advantages. Carbon ions are characterized by an highly localized dose deposition at the end of the radiation range, i.e. the Bragg peak. Moreover, they provide an enhanced relative biological effectiveness with respect to photon or proton irradiation. Thanks to these advantages, carbon beams are currently used in some hadrontherapy facilities around the world [1].

However, as carbon nuclei penetrate the human tissues, they may undergo inelastic nuclear reactions leading to the production of secondary fragments. Carbon fragmentation generates a mixed particle field, whose dose distribution is different from what would be expected in the absence of nuclear interactions. Moreover, fragments having charges and velocities different from that of the carbon beam

\footnotetext{
a e-mail: marzio.denapoli@ct.infn.it

$\mathrm{b}_{\text {deceased }}$
}

This is an Open Access article distributed under the terms of the Creative Commons Attribution License 2.0, which permits unrestricted use, distribution, and reproduction in any medium, provided the original work is properly cited. 
produce different biological effects [2]. Therefore, carbon fragmentation has to be correctly evaluated when planning a tumor treatment. The incident ions lose their energy passing through the patient's body so that the inelastic nuclear reactions may occur at energies much lower than the incident ones. The nuclear interactions of the incident ions must be taken into account not only in hadrontherapy but also in understanding and addressing the effects of the galactic cosmic rays on humans in space. One of the most serious health problems for crews of deep space missions is the radiogenic risk [3]. In order to correctly evaluate such risk, the nuclear reactions of the galactic cosmic rays inside the shielding materials of the spacecraft and the astronaut bodies must be considered. The particles and energies commonly used for hadrontherapy overlap the low end of the charge and energy range of greatest interest for space radiation applications, $Z=1-26$ and approximately $100-1000 \mathrm{MeV} /$ nucleon. In planning tumor treatments and space missions, the only way to overcome the approximations of analytical calculations in the case of mixed radiation fields and complex geometries is the use of reliable Monte Carlo codes, such as GEANT4 [4], FLUKA [5], MCNPX [6], SHIELD-HIT [7] and PHITS [8]. However, in order to precisely simulate the spatial dose distribution and the resulting biological effects in the human body, the physical models used in the Monte Carlo codes need to be tuned and validated by experimental fragmentation data. With the aim of measuring fragmentation cross sections, some dedicated experiments have been already performed at relativistic energies [9]. Since at Fermi energies only few data are available in literature, we decided to fill this gap measuring an extensive set of fragmentation cross sections in this energy regime [10]. In this paper we present original new data on the ${ }^{12} \mathrm{C}+{ }^{197} \mathrm{Au}$ fragmentation reaction, obtained in a large experimental campaign. Other results on the same target are under publication [11].

\section{Experimental Setup}

The experiment was performed at the Superconducting Cyclotron (CS) of the INFN - LNS in Catania. A ${ }^{12} \mathrm{C}$ beam was accelerated at $62 \mathrm{AMeV}$ and hit to a $104 \mu \mathrm{g} / \mathrm{cm}^{2}$ or $147 \mu \mathrm{g} / \mathrm{cm}^{2}$ thick ${ }^{12} \mathrm{C}$ or ${ }^{197} \mathrm{Au}$ target. Energy and emission angle of charged fragments $(1 \leq Z \leq 6)$ produced in the interaction with the target were measured by a detection setup consisting of two Si-CsI hodoscopes [12] (Fig. 1):

- The Hodo-Small - formed by 81 two-fold telescopes: $300 \mu \mathrm{m}$ Silicon detectors $1 \times 1 \mathrm{~cm}^{2}$ of active area followed by a $1 \times 1 \mathrm{~cm}^{2}$ and $10 \mathrm{~cm}$ long $\mathrm{CsI}(\mathrm{Tl})$;

- The Hodo-Big - formed by 88 three-fold telescopes: $50 \mu \mathrm{m}+300 \mu \mathrm{m}$ Silicon detectors both having $3 \times 3 \mathrm{~cm}^{2}$ surface followed by a $6 \mathrm{~cm}$ long $\mathrm{CsI}(\mathrm{Tl})$.

In the present experiment the Hodo-Small was placed $80 \mathrm{~cm}$ far from the target, covering the angular region $2.2^{\circ} \leq \theta_{l a b} \leq 5.5^{\circ}$. The Hodo-Big was placed at $60 \mathrm{~cm}$ from the target covering the angular region $7.6^{\circ} \leq \theta_{\text {lab }} \leq 21.8^{\circ}$. Fragments detected in each telescope were identified in charge and mass by using the $\Delta \mathrm{E}_{-} \mathrm{E}_{\text {res }}$ identification technique, being $\Delta \mathrm{E}$ and $\mathrm{E}_{\text {res }}$ the energy loss and the residual energy measured in the Si and CsI detectors, respectively. The absolute cross sections were determined from the measured fragment yields, corrected for the acquisition system dead-time, the target thickness and the beam current measured with a Faraday cup. The uncertainties in the cross sections result from the statistical errors in the fragment yields, the uncertainties on the target thickness, the number of incident ions and the dead-time.

\section{Fragmentation Cross Sections}

Figure 2 shows an example of the measured cross sections $d \sigma / d \Omega$ for the ${ }^{4} \mathrm{He},{ }^{6} \mathrm{Li},{ }^{7} \mathrm{Li}$ and ${ }^{7} \mathrm{Be}$ production in the ${ }^{12} \mathrm{C}+{ }^{197} \mathrm{Au}$ and ${ }^{12} \mathrm{C}+{ }^{12} \mathrm{C}$ reactions at $\theta_{\text {lab }}=8.6^{\circ}$. The comparison between the cross sections obtained in the two cases shows that in both reactions the different isotopes are produced with 

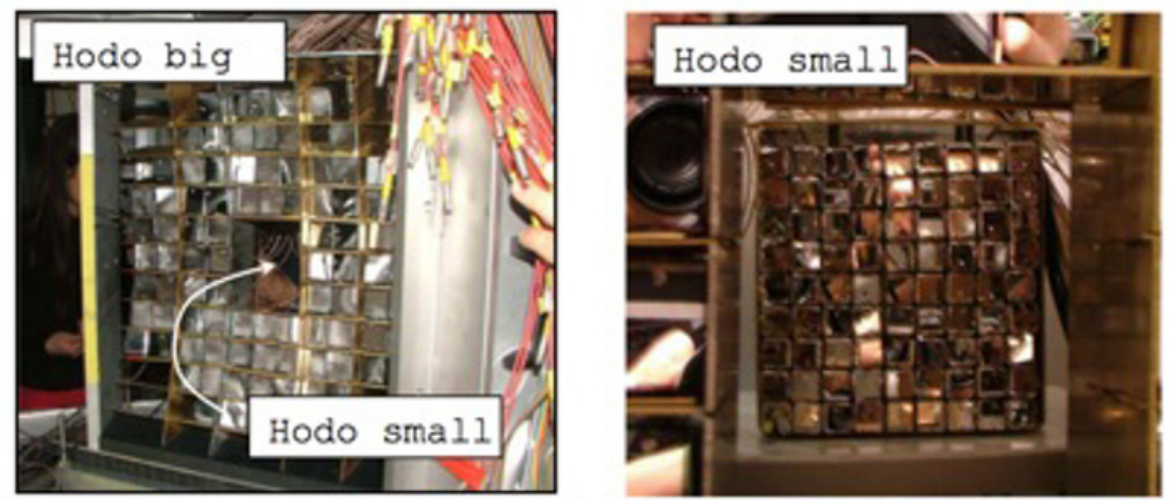

Figure 1. Pictures of the two Si-CsI hodoscopes used to detect the secondary fragments.

the same relative abundances and ${ }^{4} \mathrm{He}$ has the highest production cross-section. As is well known, because of their greater binding energy, $\alpha$ particles are very stable nuclei compared to other light elements. This aspect may lead to clustering phenomena so that ${ }^{12} \mathrm{C}$ ion can be described as $\alpha$-cluster structures and the break-up of ${ }^{12} \mathrm{C}$ into three $\alpha$ particles is extremely probable.

A strong target dependence is observed in the absolute value of the cross sections. Those measured with the ${ }^{197} \mathrm{Au}$ target are significantly higher than those measured with the ${ }^{12} \mathrm{C}$ target. This target-size effect has been already observed at relativistic energies, the so-called factorization, and is considered a basic law of the projectile fragmentation reactions [13, 14]. At Fermi energies different dissipative reaction mechanisms can contribute, together with the fragmentation one, to the fragment production at $\theta_{\text {lab }}=8.6^{\circ}$ [10]. Therefore, the observed target dependence could be also due to dissipative mechanisms since they result from more central collisions in which the target plays an important role. A detailed analysis of the fragment energy spectra and their evolution as a function of the emission angle will allow to better investigate how the different reaction mechanisms are influenced by the target.

\section{Conclusions}

The cross sections of the fragments produced in the ${ }^{12} \mathrm{C}+{ }^{12} \mathrm{C}$ and ${ }^{12} \mathrm{C}+{ }^{197} \mathrm{Au}$ reactions at $62 \mathrm{AMeV}$ have been measured at LNS. The comparison between the data measured in the two reactions shows a strong dependence of the cross sections on the target. This observation confirms the necessity to collect a wide set of fragmentation cross sections with different projectile-target and incident energy combinations. In this way it will be possible to tune the nuclear reaction models implemented in the Monte Carlo code with the prediction accuracy requested in hadrontherapy and space radiation protection.

\section{References}

[1] D. Schardt, T. Elsasser, D. Schulz-Ertner, Rev. Mod. Phys. 82, 383 (2010).

[2] Friedrich et al., Acta Oncol. 52, 589 (2013).

[3] M. Durante, F. A. Cucinotta, Rev. Mod. Phys. 83, 1245 (2011).

[4] S. Agostinelli et al., Nucl. Instrum. Methods A 506, 250 (2003). 


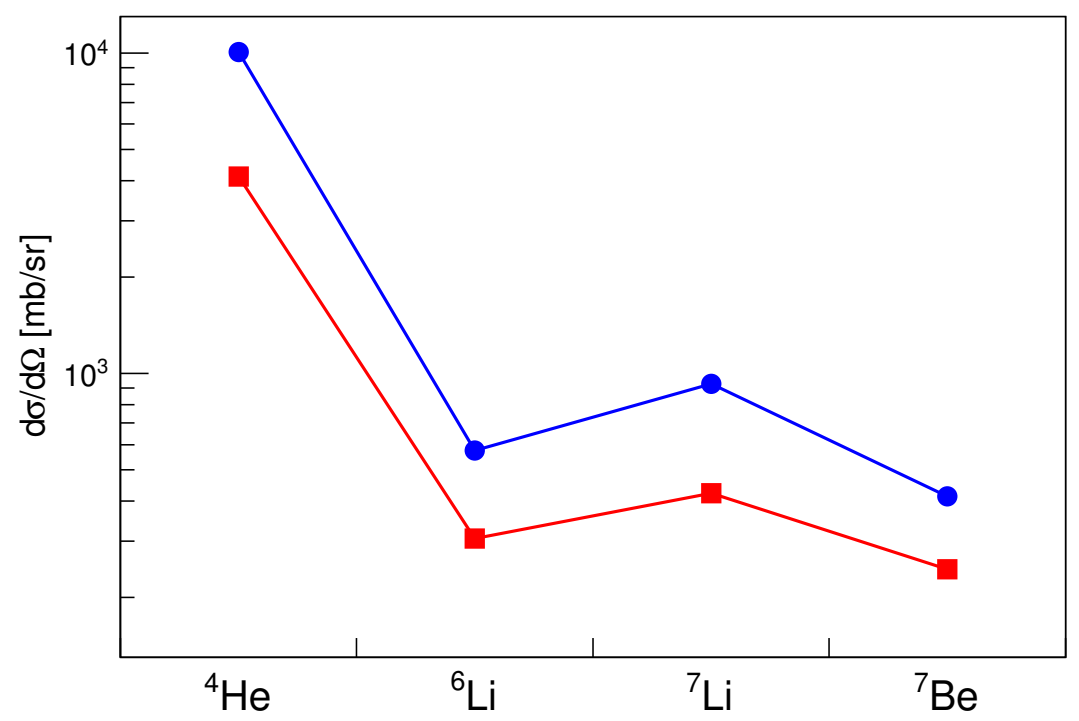

Figure 2. Experimental cross sections $d \sigma / d \Omega$ for the ${ }^{4} \mathrm{He},{ }^{6} \mathrm{Li},{ }^{7} \mathrm{Li}$ and ${ }^{7} \mathrm{Be}$ production in the ${ }^{12} \mathrm{C}+{ }^{197} \mathrm{Au}(\bullet)$ and ${ }^{12} \mathrm{C}+{ }^{12} \mathrm{C}(\mathbf{\square})$ reactions at $\theta_{\text {lab }}=8.6^{\circ}$. Error bars are smaller than the symbols size

[5] G. Battistoni et al., AIP Conf. Proc. 896, 3149 (2007).

[6] MCNPX Users Manual Version 2.4.0

[7] I. Gudowska et al., Phys. Med. Biol. 49, 193358 (2004).

[8] K. Niita et al., Radiat. Meas. 41, 108090 (2006).

[9] JW. Norbury et al., Radiat. Meas. 47, 315 (2012).

[10] M. De Napoli et al., Phys. Med. Biol. 57, 7651 (2012).

[11] M. De Napoli et al., Nuclear Data Sheets, in press.

[12] G. Raciti et al., Phys. Rev. Lett. 100, 192503 (2008).

[13] D.L. Olson et al., Phys. Rev. C 28, 1602 (1983).

[14] J.R. Cummings Olson et al., Phys. Rev. C 42, 2530 (1990). 